\title{
Prediction of Metastasis and Recurrence in Colorectal Cancer Based on Gene Expression Analysis: Ready for the Clinic?
}

\author{
Masaki Shibayama ${ }^{1}$, Matthias Maak ${ }^{2}$, Ulrich Nitsche ${ }^{2}$, Kengo Gotoh ${ }^{1}$, Robert Rosenberg ${ }^{2}$ \\ and Klaus-Peter Janssen ${ }^{2}$ * \\ 1 Sysmex Corporation, Central Research Laboratories, Kobe 651-2271, Japan; \\ E-Mail: Shibayama.Masaki@ sysmex.co.jp (M.S.); Gotoh.Kengo@ sysmex.co.jp (K.G.) \\ 2 Chirurgische Klinik, Klinikum Rechts der Isar der TUM, München 81657, Germany; \\ E-Mails: maak@chir.med.tu-muenchen.de (M.M.); nitsche@ chir.med.tu-muenchen.de (U.N.); \\ rosenberg@chir.med.tu-muenchen.de (R.R.) \\ * Author to whom correspondence should be addressed; E-Mail: klaus-peter.janssen@1rz.tum.de; \\ Tel.: +49-89-4140-2066; Fax: +49-89-4140-6031.
}

Received: 10 May 2011; in revised form: 23 June 2011 / Accepted: 27 June 2011 /

Published: 7 July 2011

\begin{abstract}
Cancers of the colon and rectum, which rank among the most frequent human tumors, are currently treated by surgical resection in locally restricted tumor stages. However, disease recurrence and formation of local and distant metastasis frequently occur even in cases with successful curative resection of the primary tumor (R0). Recent technological advances in molecular diagnostic analysis have led to a wealth of knowledge about the changes in gene transcription in all stages of colorectal tumors. Differential gene expression, or transcriptome analysis, has been proposed by many groups to predict disease recurrence, clinical outcome, and also response to therapy, in addition to the wellestablished clinico-pathological factors. However, the clinical usability of gene expression profiling as a reliable and robust prognostic tool that allows evidence-based clinical decisions is currently under debate. In this review, we will discuss the most recent data on the prognostic significance and potential clinical application of genome wide expression analysis in colorectal cancer.
\end{abstract}

Keywords: colorectal cancer; transcriptome; metastasis; prognosis; therapy prediction 
Abbreviations: T-based: tumor based; NM-based: non-neoplastic mucosa based; A-based: adjacent non-neoplastic mucosa based gene signature; NM: non-neoplastic mucosa; CRC: colorectal cancer; CC: colon cancer

\section{Introduction}

Colorectal cancer (CRC) is one of the most common cancers and the second leading cause of cancer-related death worldwide [1,2]. The progression of colorectal cancer is well documented in histopathological terms, and the molecular genetic changes associated with the so-called "adenoma-carcinoma" tumor progression sequence have been studied extensively over the last decades. Several types of staging systems have been developed for colorectal cancer, such as the Dukes' system, the modified Astler-Coller staging system and the TNM system introduced by the American Joint Committee on Cancer (AJCC) and the International Union Against Cancer (UICC) [3-6]. These staging systems rely on the size and extent of the primary tumor, on the metastatic spread to lymph nodes and distant organ sites, and on lymphatic and vascular invasion (TNM system). Metastasis formation is the major cause of death in patients with colorectal cancer, and depending on tumor stage, liver metastases occur in $20 \%$ to $70 \%$ of patients, and lung metastases in $10 \%$ to $20 \%$ of cases. For patients with locally restricted colon tumors without lymph node metastasis, surgical tumor resection is the current standard therapy. Adjuvant chemotherapy (radio-chemotherapy in the case of rectal cancer) is generally only recommended for more advanced tumor stages. However, all current staging systems have their shortcomings and limitations. To state an example of high clinical relevance: it is not possible to prospectively identify the high-risk group of 20-30\% of patients with locally restricted stage II (UICC) colon cancer that will suffer from disease recurrence. This group of patients may actually benefit from adjuvant therapy in addition to surgical treatment, even though chemotherapy is not recommended according to current guidelines [7].

Recently, increasing knowledge of the molecular etiology of tumor progression from adenoma to adenocarcinoma of the colon has facilitated the identification of a number of prognostic and predictive biomarker candidates. A sequential process of epigenetic alterations and gene mutations is widely believed to drive this process, and the acquisition of these mutations is facilitated by the loss of genomic stability, which occurs in two major forms: as chromosomal instability (CIN), and, less frequently, as microsatellite instability (MSI). A relatively small number of signaling pathways seems to drive the progression of colorectal cancer, since aberrant activation of the canonical WNT-pathway, mutations in the oncogene KRAS and the tumor suppressor TP53 can be found in the majority of all cases of sporadic colorectal cancer. However, next to these essential "driver" mutations, a multitude of "passenger" mutations or epigenetic alterations are likely to occur, contributing to heterogeneity. The clinical usefulness of detecting oncogenic KRAS mutations, mutations and loss of p53 function, or loss of heterozygosity of chromosome18q, microsatellite and chromosomal instability status have been intensively investigated in the light of their prognostic capacity. Despite these enormous efforts aimed at finding molecular markers for individualized medicine, the ASCO 2006 guidelines for use of tumor markers in colorectal cancer did not conclude that there was sufficient data to support the use of any of 
these markers [8]. However, the use of KRAS mutations to predict the efficacy of a therapy directed against the EGFR (epidermal growth factor receptor) has been well documented [9].

DNA microarray technology, the so-called "gene chips", is one of the comprehensive or "omics" approaches which allows the concomitant profiling of the expression of thousands of genes, reflecting the global transcription activity at one given point in time. This approach has been widely used in medical oncology, with a good potential to provide a detailed and comprehensive view of gene expression changes involved in tumor initiation and progression. Moreover, this method holds the promise to determine the sensitivity towards classical cytotoxic chemo/radio-therapies, or the new class of "biological" like the anti-EGFR therapy, and allows an insight into the underlying resistance mechanisms. However, even though much has been learned about cancer biology from transcriptome profiling of normal colon mucosa as compared to carcinoma tissue, the clinical application of microarray technology is currently very limited in gastrointestinal oncology. If the hypothesis is correct that a limited number of critical "driver" pathways determine clinical outcome in most cancers, the results from transcriptome studies should identify highly similar subsets of transcripts. As an example, the WNT-pathway is activated by mutations in the APC tumor suppressor (adenomatous polyposis coli) in the majority of colorectal cancers [10]. Therefore, target genes of the WNT-pathway should be found to be up-regulated in transcriptome studies. Moreover, specific gene expression changes associated with good or bad prognosis should occur in most studies, as a result of the similar biology that underlies the processes of tumor progression and metastasis. To our great surprise, this was not the case. In fact, the overlap between published gene expression signatures with prognostic relevance for colorectal cancer was very small. This raises questions about the genetic and epigenetic heterogeneity inherent to colorectal cancer, and about the usefulness and stringency of new candidate biomarkers. This review addresses the question whether the current literature reflects the application of gene expression profiling for individualized treatment decisions. In other words, if a clinical practitioner in gastrointestinal oncology or surgery would actually base a therapy decision on the results of a gene expression test for a given patient. Taken together, our aim was to analyze and review reported gene signatures obtained for human colorectal cancer from the viewpoints of tumor biology and clinical practice.

\section{Results and Discussion}

\subsection{Overview-Proposed Prognostic Gene-Signatures for Early Stage Colorectal Cancer}

The focus of our analysis was to investigate biological features related to patient prognosis commonly observed in previous transcriptome studies. Therefore, we reviewed publications between January 2001 and August 2010, in which patient samples from early stage colorectal cancer (UICC stage I-III) were examined by microarray-based gene expression analysis. For that purpose, the PubMed database (http://www.ncbi.nlm.nih.gov/pubmed/) was searched with the keywords 'gene expression, array, or profiling', and 'cancer, tumor, carcinoma, or adenocarcinoma', and 'colon, rectal, or colorectal', and 85 publications were thus selected for this review. In addition, three reports focusing on prognostic markers of colorectal cancer which were previously reviewed by Nannini et al. [11] were included in the list. Among the 88 publications, ten are review articles, three are meta-analysis reports 
and 75 are original research articles (see Table 1). Among the latter 75, the CGH (complete genomic hybridization) or "Exon array" method (Genomic analysis) was used in 12 cases, mRNA-based approaches (cDNA or oligonucleotide microarrays, serial analysis of gene expression / SAGE, cDNA macroarray and low density arrays / LDA) were performed in the remaining 63 studies. In the present review, we focused on original studies on colorectal cancer investigating the mRNA based gene-expression signature with a special emphasis on the prognosis, and not on the expression differences between various cancer stages, or between carcinoma and normal tissue. In 15 articles, candidate mRNA expression signatures were proposed to predict colorectal cancer recurrence (Table 2). The respective study designs, such as the chosen platform of microarray technology, the tumor stage of the patients, and the number of genes of the identified prognostic signature for the recurrence prediction, are shown in Table 2. Since the technical background (e.g., type of microarray technology) and the analytical strategies (as discussed below) vary greatly, a stringent meta-analysis was not appropriate. Instead, we focused on the extraction and identification of the individual genes that were consistently reported in the original studies, according to our hypothesis of common conserved "driver" mutations. In 12 of the 15 studies, prognostic gene expression signatures were identified based on colorectal tumor samples, whereas Barrier et al. identified signatures based on the analysis of non-neoplastic mucosa tissue obtained from patients with colorectal cancer CRC (three studies in total). Actually, less than a total of 50 individual gene transcripts were selected as prognostic signature in eight of the studies. To investigate if biological features were consistently observed in these eight studies (i.e., the driver mutations leading to conserved changes in gene transcription), the gene expression signatures were analyzed for overlaps. When the eight independent sets of gene expression were compared, only one gene (APOC1) was identified in more than two independent studies (study No.10 and No.13, Table 2). Interestingly, the other six prognostic expression signatures did not contain the gene APOC1. This unexpected result reveals surprisingly low consistency among the prognostic gene signatures from different studies. The APOC1 gene maps to chromosome 19, it encodes the gene product apolipoprotein C-I which is mainly expressed in the liver, but also has been reported as potential serum biomarker on in colorectal, breast and pancreatic cancer [12-14]. Apolipoprotein C-I has anti-apoptotic and proliferation-enhancing effects on pancreatic cancer cells [15].

Table 1. Overview: selected literature on microarrays.

\begin{tabular}{lll}
\hline Articles Examined & & 88 \\
\hline & Reviews & 10 \\
& Meta-analysis & 3 \\
& Research article & 75 \\
\hline Research article & & \\
\hline & Genomic analysis & 12 \\
& mRNA expression profile & 63 \\
\hline
\end{tabular}


Table 2. Literature on microarrays - sorted by approach, number of cases, and tissue type analyzed.

\begin{tabular}{|c|c|c|c|c|c|c|}
\hline No & First Author & Year & Platform & $\begin{array}{l}\text { Tumor Stages/Patient } \\
\text { Cohort Analyzed }\end{array}$ & $\begin{array}{l}\text { Number of Genes in } \\
\text { the Prognostic } \\
\text { Expression Signature }\end{array}$ & Tissue \\
\hline 1 & Yoshida & 2010 & oligonucleotide & Stage I-IV CRC & 4 genes & tumor \\
\hline 2 & Jorissen & 2009 & oligonucleotide & Dukes' B/C CRC & 128 genes & tumor \\
\hline 3 & Cavalieri & 2007 & cDNA & CRC of all stage & 8 genes & tumor \\
\hline 4 & Lin & 2007 & oligonucleotide & $\begin{array}{l}\text { CRC of all stages (NZ) / } \\
\text { Stage I/II CRC (GE) }\end{array}$ & $\begin{array}{l}22 \text { genes }(\mathrm{NZ}) / \\
19 \text { genes }(\mathrm{GE})\end{array}$ & tumor \\
\hline 5 & Yamasaki & 2007 & cDNA & Stage II/III CRC & 119 genes & tumor \\
\hline 6 & Barrier & 2007 & oligonucleotide & Stage II CC & 70 genes (NM-based) & NM \\
\hline 7 & Bianchini [35] & 2006 & cDNA & \multicolumn{2}{|c|}{ Stage I-III CRC (Dukes' B/C) 88 genes } & tumor \\
\hline 8 & Barrier & 2006 & oligonucleotide & Stage II CC & 30 genes & tumor \\
\hline 9 & Arango [36]. & 2005 & oligonucleotide & Dukes' C CC & $\begin{array}{l}\text { 5-nearest neighbors } \\
\text { and } 17 \text { genes }\end{array}$ & tumor \\
\hline 10 & Eschrich [37]. & 2005 & cDNA & Dukes' stage B-D CRC & 43 genes & tumor \\
\hline 11 & Barrier & 2005 & oligonucleotide & Stage II/III CC & 47 genes (A-based) & NM \\
\hline 12 & Barrier & 2005 & oligonucleotide & Stage II/III CC & $\begin{array}{l}30 \text { genes (T-based), } 70 \\
\text { genes (NM-based) }\end{array}$ & $\begin{array}{l}\text { tumor,N } \\
\mathrm{M}\end{array}$ \\
\hline 13 & Wang & 2004 & oligonucleotide & Dukes' B CC & 23 genes & tumor \\
\hline 14 & Bertucci [38]. & 2004 & cDNA & Stage I-III CRC & 244 cDNA clones & tumor \\
\hline 15 & Ramaswamy & 2003 & oligonucleotide & $\begin{array}{l}\text { lung, breast, prostate, } \\
\text { colorectal, uterus, ovary }\end{array}$ & 17 genes & tumor \\
\hline
\end{tabular}

\subsection{Inconsistencies in Gene Signatures between Validated Studies}

Even though the prognostic gene expression signatures analyzed here varied greatly and showed no major consistency among them, this does not imply that all prognostic signatures are unreliable. Actually, some of the signatures have been successfully validated. Wang et al. performed a microarray analysis using samples from $n=74$ UICC II patients, and proposed a candidate 23-gene signature that was associated with disease recurrence [16]. Independently, Barrier et al. evaluated this 23-gene expression signature for $\mathrm{n}=50$ patients with stage II (UICC) colorectal cancer. Importantly, it was reported that the 23-gene signature led to a fairly accurate prediction for the prognosis (overall mean accuracy of 67.1\%) [17]. To the best of our knowledge, this was the first report for a prognostic gene signature for colorectal cancer which was successfully validated by an independent research group. Interestingly, Barrier et al. developed their own prognostic gene expression signature with 30 completely independent genes using exactly the same patient cohort. This suggests the possibility to develop more than one valid gene expression signature for the prognosis of patients with early stage colorectal cancer. Colorectal cancer is thought to develop over years and decades from early precursor lesions, accompanied by a plethora of epigenetic and genetic alterations. These alterations may not only vary between individual patients, but even within one particular cancer, and thus contribute to expression heterogeneity in colorectal cancer. In addition to this inherent biological "noise", one has to deal in the clinical context with technical variation, population differences, and the requirement of excellent mRNA conservation in a clinically resected tumor specimen. 
However, the central hallmarks of cancer, as defined by Weinberg and Hanahan, require certain pathways to be altered, otherwise the tumor will fail to grow and progress [18]. These changes are certainly linked to expression differences, and it is therefore likely that, even though heterogeneous gene expression renders the task difficult, valuable prognostic information can be derived from transcriptome data sets. A large, yet limited number of completely different prognostic "gene expression signatures" may exist that allow the stratification of patients.

In accordance, our group has obtained similar observations [19,20]. In a cooperation between surgical centers in New Zealand and Germany, $\mathrm{n}=147$ patients from New Zealand (UICC stage I-IV), and $\mathrm{n}=55$ German patients (UICC stage I and II) were investigated. Two independent platforms were applied, using oligonucleotide printed microarrays for the New Zealand samples and Affymetrix microarrays for the samples from Germany. This approach yielded a 22-gene prognostic expression set from the New Zealand cohort, and a 19-gene expression set from the German cohort. Importantly, in spite of the differences in the technology and in the clinical background of the cohorts, both prognostic candidate gene signatures retained prognostic power when applied to the alternate series of patients, in a so-called "cross-validation" approach. However, similar to the findings discussed above, both "prognostic expression signatures" were mutually exclusive and contained just one overlapping transcript, the gene TOPK (T-LAK cell-originated protein kinase, or PDZ-binding kinase). The gene TOPK has been assigned to chromosome 8 , it encodes a protein kinase which is mainly expressed in testis and cells of the lymphoid lineage [21]. It has recently been shown that the TOPK kinase plays a role during mitosis in the DNA damage response, and directly interacts on the protein level with the tumor suppressor p53 [22]. Thus, it seems evident that there is to date no unique gene expression signature that would represent the best or "exclusive" set of genes for predicting disease recurrence, even based on independently cross-validated studies. Rather, it could be expected that a comprehensive approach that takes multiple independent gene expression signatures into account may yield a robust prognostic tool with the sensitivity and specificity levels required for actual clinical needs.

\subsection{Strategies for the Development of Clinically Useful Prognostic Gene Signatures}

The surprisingly low consistency between independently published prognostic gene expression signatures may be caused by different set-ups in the strategies of the studies. Two quite different approaches are usually applied to place patients into two groups, in order to develop prognostic gene expression signatures with the ultimate goal of distinguishing patients with good and poor prognosis.

The first approach is to investigate the putative molecular nature of eventual metastasis based on the primary tumor itself. Since tumor-related death is in most cases attributable to metastasis, as mentioned by Ramaswamy et al. [23], patients with "metastasis-like" gene expression signatures in the primary tumor are likely to have a significantly worse clinical outcome. As stated earlier, metastatic spread in colorectal cancer is observed frequently in the liver, even though many other sites like the lung, brain and peritoneal cavity can be affected as well. The local microenvironment of these different compartments varies greatly in terms of extracellular matrix composition and local growth factor production. In the case of breast cancer, it has been proposed that glial cells produce cytokines which contribute in a paracrine way to the formation of brain metastasis [24]. However, it is still unclear which molecular mechanisms determine the site of distant metastasis in colon cancer. Ramaswamy et al. 
identified the expression profiles of 17 genes that distinguish primary and metastatic adenocarcinoma, based on the analysis of metastatic and primary tumor tissue of diverse origin, such as lung, breast, prostate, large intestine, uterus and ovary [23]. Indeed, tumors bearing a "metastasis-like" gene-expression signature at diagnosis were more likely to develop metachronous distant metastases. However, it should be noticed here that a considerable proportion of the refined gene-expression signature associated with metastasis seems to be derived from non-epithelial stroma components of the tumor. Therefore, RNA extraction from microdissected samples as opposed to non-dissected tumor samples, which may contain considerable stroma components, may also contribute to heterogeneity.

In a similar approach, Yamasaki and co-workers analyzed gene expression profiles of $\mathrm{n}=104$ samples corresponding to the oncogenic development in an assumed chronological order, including normal mucosa, locally restricted and metastasized primary tumors, and liver metastasis [24]. Transcripts were classified into four distinct groups: (A) genes differentially expressed during all tumor stages, (B) genes differentially expressed in synchronous or metachronous metastasized primary tumors and liver metastasis, (C) genes differentially expressed specifically in liver metastasis, and (D) genes that were not characterized by recognizable expression patterns at all steps of cancer development [24]. It was found that a set of 119 genes of type B allowed the classification of tumors into two classes, the "localized" and the "metastasized" class. Importantly, the disease-free survival and overall survival were significantly longer in the "localized" class than the "metastasized" class [24].

The second approach is to directly compare differential gene expression between patients from similar tumor stages but with good or poor prognosis. Jorissen and co-workers directly focused on the clinical tumor stage according to Dukes' staging system, and were able to determine 128 genes which showed reproducible expression change between Dukes stage A and D cancer [25]. Using this gene signature, the intermediate-stage cancers (stage B and C) were classified into "stage A-like/good prognosis" or "stage D-like/poor prognosis" subtypes [25]. The treatment-adjusted hazard ratio for the recurrence in "stage D-like" cancers was 10.3 for stage B patients, and 2.9 for stage C patients, respectively. Thus, metastasis-associated gene expression changes can be used to refine the traditional outcome prediction which is mainly based on the histopathological TNM-staging system.

Finally, differences in statistical analysis methods may yet be another possible reason for the low consistency between gene signatures from different groups. Cavalieri et al. compared three frequently used statistical methods: the significant analysis of microarray (SAM) tool, the Trend Filter tool, and Cox's proportional Hazard Model [26]. SAM is specified as a "one-class-method", which tests the hypothesis of over- or under-expression for every gene relative to the reference gene, controlling for the false discovery rate (FDR). The Trend Filter tool was derived from the Trend Capture feature of the commercial package "Rosetta Resolver" developed by Rosetta Biosoftware. In the routine Trend Filter tool, a gene is said to exhibit a "trend" in an experimental group if it is over- or under-expressed in a set proportion of the patients in the group. Conversely, a gene does not exhibit a "trend", if its over- or under-expression is limited to a small proportion of the patients in the group. Finally, the widely used Cox's Hazard model assumes that the underlying hazard rate is a function of several independent variables. In the model used by Cavalieri and co-workers, the parameters age, sex, Dukes' stage and tumor localization were considered as covariates, and one regression coefficient for each gene was obtained [26]. Next, a probabilistic clustering algorithm was performed, and the genes grouped in the 
more extreme clusters were considered. With this approach, two groups of genes were identified grouped in the highest and in the lowest clusters. As a result, seven genes were retained as prognostic markers by both SAM and Cox Hazard analysis, and only a single gene was commonly retained by the Cox Hazard model, and the Trend Filter analysis [26]. Thus, different statistical methodology may contribute to greatly varied gene expression signatures.

\subsection{Heterogeneity in Colorectal Cancer Tissue-Stroma Contributions}

Great interest has been shown in the biological characterization of the identified genes from the various individual signatures. For example, activity variations of metabolic pathways could be identified through the study of the expression of genes attributed to these pathways in public database (KEGG, Reactome, GenMAPP). A comprehensive pathway analysis showed that patients with favorable prognosis had several activated metabolic pathways, including carbon metabolism, transcription, amino acid and nitrogen metabolism, signaling and fibroblast growth factor receptor pathways. [26]. Moreover, the cell-biological heterogeneity of colorectal cancer is another crucial aspect to be considered for prognostic gene signatures, especially for studies that originate from nonmicrodissected tumor specimens. We could show that a significant part of the prognostic gene expression signature in colon cancer concerned genes involved in immune modulation, such as chemotaxis-inducing cytokines which regulate the tumor stroma interaction, e.g., by attracting T-cells or modulating angiogenesis $[19,27]$. There is some evidence that interactions between stromal and cancer cells are a prerequisite for metastases to occur [28,29]. Though it remains unclear whether this metastatic potential originates in cancer cells and/or in stroma compartments, it may be present from the start of the tumor $[23,30]$. Accepting this theory, the non-neoplastic, normally appearing mucosa on which the tumor has arisen may contain some helpful information. Barrier et al. compared gene expression profiles of tumor samples and adjacent non-neoplastic colon mucosa. Three datasets were generated, including the gene expression ratios in tumor samples $(\mathrm{T})$, in adjacent non-neoplastic mucosa samples (A), and the log-ratio of the gene expression measures (L) [31]. Unexpectedly, A-based predictors were more stable (i.e., less sensitive to changes of parameters, such as number of genes and neighbors) than $\mathrm{T}$ - or L-based predictors, suggesting the potential usefulness of the non-neoplastic mucosa in predicting the prognosis of colon cancer patients with stage II and III tumors. In addition, the same group further assessed the possibility of developing a prognostic gene signature [17]. Adjacent non-neoplastic colon mucosa samples were collected at a distance between 5 and $10 \mathrm{~cm}$ from the gross tumor limit at the time of surgery, and a 70-gene expression signature was developed based on microarray gene expression measures. Interestingly, in contrast to tumor-based gene signature, the non-neoplastic mucosa-based gene signature was suggested to be more specific than sensitive, that is, more able to detect patients with a good prognosis.

Thus, the heterogeneity in colorectal cancer undoubtedly arises in part by varying stroma contributions. However, genetic heterogeneity between the cancer cells themselves may additionally increase the difficulty in developing prognostic gene signature. Baisse and co-workers reported an intratumoral genetic heterogeneity for advanced sporadic colorectal carcinoma [32]. It has been found that more than $60 \%$ of the analyzed tumors were heterogeneous for at least one genetic alteration. Actually, intratumoral heterogeneity was more frequently observed in the form of LOH (loss of 
heterozygosity) than in the form of point mutations. Notably, 67\% and 58\% of LOH events were heterogeneous at the APC (adenomatous polyposis coli) and DCC (deleted in colon cancer) tumor suppressor gene loci, respectively, and $20 \%$ of point mutation of either the KRAS or TP53 gene were observed to be heterogeneous. Consistent with these findings, it has been pointed out that there are higher "noise" levels in the expression data from colorectal cancer than in those of other solid cancers [33]. To perform a more reliable expression profiling analysis, Yoshida et al. combined the analysis of DNA copy number aberration with a comprehensive gene expression screening using $\mathrm{n}=79$ cases of colorectal cancer (UICC stages I to IV) [33]. Since chromosomal aberrations and genomic instability are often observed in sporadic colorectal cancer, this impressive effort seems well justified. This approach led to the identification of four genes associated with tumor recurrence, four further genes associated with node status and one gene associated with distant metastasis. High expression level and copy number gain of the S100 calcium binding protein A2 gene (S100A2), abhydrolase domain containing 2 (ABHD2), as well as low expression level and copy number losses of ABHD12 (abhydrolase domain containing 12), and the oncoprotein induced transcript 3 (OIT3) were found in recurrent cases compared with non-recurrent cases [33]. This combined analysis of copy-number analysis and gene expression profiling could be helpful in understanding the clinic-pathological meaning of genomic instability.

\section{Conclusions}

Microarray-based classifiers for cancer prognosis have become statistically highly reliable, as mentioned by Fan et al. [34]. Several prognostic gene expression signatures have been successfully developed so far for colorectal cancer, and independent validation could be obtained in some cases. However, there is surprisingly little overlap in the gene expression signatures published so far. The low consistency between the different studies may be in part attributed to methodological and technical variances, but it seems evident that colorectal cancer is an extremely heterogeneous disease at the molecular level. This precludes a wide-spread use of expression profiling for clinical purposes, and a selection of an ideal and personalized therapy regime based on gene expression signatures remains elusive. Therefore, it seems evident that further multicenter study and standardized meta-analysis are required. Moreover, currently developed next-generation sequencing approaches may provide additional prognostic information which may not be obtained only by transcriptome profiling. Nevertheless, from a biological point of view, the reported gene expression signatures have largely contributed to our current understanding of tumor initiation and progression in the large intestine.

\section{References}

1. Jemal, A.; Bray, F.; Center, M.M.; Ferlay, J.; Ward, E.; Forman, D. Global cancer statistics. $C A$ Cancer J. Clin. 2011, 61, 69-90.

2. Ferlay, J.; Shin, H.R.; Bray, F.; Forman, D.; Mathers, C.; Parkin, D.M. Estimates of worldwide burden of cancer in 2008: GLOBOCAN 2008. Int. J. Cancer 2010, 127, 2893-2917

3. Markowitz, S.D.; Bertagnolli, M.M. Molecular origins of cancer: Molecular basis of colorectal cancer. N. Engl. J. Med. 2009, 361, 2449-2460. 
4. Astler, V.B.; Coller, F.A. The prognostic significance of direct extension of carcinoma of the colon and rectum. Ann. Surg. 1954, 139, 846-852.

5. O'Connell, J.B.; Maggard, M.A.; Ko, C.Y. Colon cancer survival rates with the new American Joint Committee on Cancer sixth edition staging. J. Natl. Cancer Inst. 2004, 96, 1420-1425.

6. Gunderson, L.L.; Jessup, J.M.; Sargent, D.J.; Greene, F.L.; Stewart, A.K. Revised TN categorization for colon cancer based on national survival outcomes data. J. Clin. Oncol. 2010, 28, 264-271.

7. Schmiegel, W.; Pox, C.; Reinacher-Schick, A.; Adler, G.; Arnold, D.; Fleig, W.; Fölsch, U.R.; Frühmorgen, P; Graeven, U.; Heinemann, V.; Hohenberger, W.; Holstege, A.; Junginger, T.; Kopp, I.; Kühlbacher, T.; Porschen, R.; Propping, P; Riemann, JF; Rödel, C; Sauer, R; Sauerbruch, T.; Schmitt, W.; Schmoll, H.J.; Seufferlein, T.; Zeitz, M.; Selbmann, H.K.; Federal Committee of Physicians and Health Insurers. S3 guidelines for colorectal carcinoma: results of an evidence-based consensus conference on February 6/7, 2004 and June 8/9, 2007 (for the topics IV, VI and VII). Z. Gastroenterol. 2010, 48, 65-136.

8. Locker, G.Y.; Hamilton, S.; Harris, J.; Jessup, J.M.; Kemeny, N.; MacDonald, J.S.; Somerfield, M.R.; Hayes, D.F.; Bast, R.C., Jr. ASCO 2006 update of recommendations for the use of tumor markers in gastrointestinal cancer. J. Clin. Oncol. 2006, 24, 5313-5327.

9. Heinemann, V.; Stintzing, S.; Kirchner, T.; Boeck, S.; Jung, A. Clinical relevance of EGFR- and KRAS-status in colorectal cancer patients treated with monoclonal antibodies directed against the EGFR. Cancer Treat. Rev. 2009, 35, 262-271

10. Fre, S.; Vignjevic, D.; Schoumacher, M.; Duffy, S.L.; Janssen, K.P.; Robine, S.; Louvard, D. Epithelial morphogenesis and intestinal cancer: New insights in signaling mechanisms. Adv. Cancer Res. 2008, 100, 85-111.

11. Nannini, M.; Pantaleo, M.A.; Maleddu, A.; Astolfi, A.; Formica, S.; Biasco, G. Gene expression profiling in colorectal cancer using microarray technologies: Results and perspectives. Cancer Treat. Rev. 2009, 35, 201-209.

12. Engwegen, J.Y.; Helgason, H.H.; Cats, A.; Harris, N.; Bonfrer, J.M.; Schellens, J.H.; Beijnen, J.H. Identification of serum proteins discriminating colorectal cancer patients and healthy controls using surface-enhanced laser desorption ionisation-time of flight mass spectrometry. World J. Gastroenterol. 2006, 12, 1536-1544.

13. Xue, A.; Scarlett, C.J.; Chung L.; Butturini G.; Scarpa A.; Gandy R.; Wilson S.R.; Baxter R.C.; Smith R.C. Discovery of serum biomarkers for pancreatic adenocarcinoma using proteomic analysis. Br. J. Cancer 2010, 103, 391-400.

14. Fan, Y.; Wang, J.; Yang, Y.; Liu, Q.; Fan,Y.; Yu, J.; Zheng, S.; Li, M.; Wang, J. Detection and identification of potential biomarkers of breast cancer. J. Cancer Res. Clin. Oncol. 2010, 136, 1243-1254.

15. Takano, S.; Yoshitomi, H.; Togawa A.; Sogawa K.; Shida T.; Kimura F.; Shimizu H.; Tomonaga T.; Nomura F.; Miyazaki, M. Apolipoprotein C-1 maintains cell survival by preventing from apoptosis in pancreatic cancer cells. Oncogene 2008, 27, 2810-2822. 
16. Wang, Y.; Jatkoe, T.; Zhang, Y.; Mutch, M.G.; Talantov, D.; Jiang, J.; McLeod, H.L.; Atkins, D. Gene expression profiles and molecular markers to predict recurrence of Dukes' B colon cancer. $J$. Clin. Oncol. 2004, 22, 1564-1571.

17. Barrier, A.; Boelle, P.Y.; Roser, F.; Gregg, J.; Tse, C.; Brault, D.; Lacaine, F.; Houry, S.; Huguier, M.; Franc, B.; Flahault, A.; Lemoine, A.; Dudoit, S. Stage II colon cancer prognosis prediction by tumor gene expression profiling. J. Clin. Oncol. 2006, 24, 4685-4691.

18. Hanahan, D.; Weinberg, R.A. Hallmarks of cancer: the next generation. Cell 2011, 144, 646-674.

19. Lin, Y.H.; Friederichs, J.; Black, M.A.; Mages, J.; Rosenberg, R.; Guilford, P.J.; Phillips, V.; Thompson-Fawcett, M.; Kasabov, N.; Toro, T.; Merrie, A.E.; van Rij, A.; Yoon, H.S.; McCall, J.L.; Siewert, J.R.; Holzmann, B.; Reeve, A.E. Multiple gene expression classifiers from different array platforms predict poor prognosis of colorectal cancer. Clin. Cancer Res. 2007, 13, 498-507.

20. Friederichs, J.; Rosenberg, R.; Mages, J.; Janssen, K.P.; Maeckl, C.; Nekarda, H.; Holzmann, B.; Siewert, J.R. Gene expression profiles of different clinical stages of colorectal carcinoma: toward a molecular genetic understanding of tumor progression. Int. J. Colorectal Dis. 2005, 20, 391-402.

21. Park, J.H.; Lin, M.L.; Nishidate, T.; Nakamura, Y.; Katagiri, T. PDZ-binding kinase/T-LAK celloriginated protein kinase; a putative cancer/testis antigen with an oncogenic activity in breast cancer. Cancer Res. 2006, 66, 9186-9195.

22. Nandi, A.K.; Ford, T.; Fleksher, D.; Neuman, B.; Rapoport, A.P. Attenuation of DNA damage checkpoint by $\mathrm{PBK}$; a novel mitotic kinase; involves protein-protein interaction with tumor suppressor p53. Biochem. Biophys. Res. Commun. 2007, 358, 181-188.

23. Ramaswamy, S.; Ross, K.N.; Lander, E.S.; Golub, T.R. A molecular signature of metastasis in primary solid tumors. Nat. Genet. 2003, 33, 49-54.

24. Yamasaki, M.; Takemasa, I.; Komori, T.; Watanabe, S.; Sekimoto, M.; Doki, Y.; Matsubara, K.; Monden, M. The gene expression profile represents the molecular nature of liver metastasis in colorectal cancer. Int. J. Oncol. 2007, 30, 129-138.

25. Jorissen, R.N.; Gibbs, P.; Christie, M.; Prakash, S.; Lipton, L.; Desai, J.; Kerr, D.; Aaltonen, L.A.; Arango, D.; Kruhoffer, M.; Orntoft, T.F.; Andersen, C.L.; Gruidl, M.; Kamath, V.P.; Eschrich, S.; Yeatman, T.J.; Sieber, O.M. Metastasis-Associated Gene Expression Changes Predict Poor Outcomes in Patients with Dukes Stage B and C Colorectal Cancer. Clin. Cancer Res. 2009, 15, 7642-7651.

26. Cavalieri, D.; Dolara, P.; Mini, E.; Luceri, C.; Castagnini, C.; Toti, S.; Maciag, K.; De Filippo, C.; Nobili, S.; Morganti, M.; Napoli, C.; Tonini, G.; Baccini, M.; Biggeri, A.; Tonelli, F.; Valanzano, R.; Orlando, C.; Gelmini, S.; Cianchi, F.; Messerini, L.; Luzzatto, L. Analysis of gene expression profiles reveals novel correlations with the clinical course of colorectal cancer. Oncol. Res. 2007, $16,535-548$.

27. Doll, D.; Keller, L.; Maak, M.; Boulesteix, A.L.; Siewert, J.R.; Holzmann, B.; Janssen, K.P. Differential expression of the chemokines GRO-2, GRO-3, and interleukin-8 in colon cancer and their impact on metastatic disease and survival. Int. J. Colorectal. Dis. 2010, 25, 573-581.

28. Barrier, A.; Roser, F.; Boelle, P.Y.; Franc, B; Tse, C; Brault, D; Lacaine, F; Houry, S; Callard, P; Penna, C; Debuire, B; Flahault, A.; Dudoit, S.; Lemoine, A. Prognosis of stage II colon cancer by non-neoplastic mucosa gene expression profiling. Oncogene 2007, 26, 2642-2648. 
29. Mueller, M.M.; Fusenig, N.E. Friends or foes - bipolar effects of the tumour stroma in cancer. Nat. Rev. Cancer 2004, 4, 839-849.

30. Bernards, R.; Weinberg, R.A. A progression puzzle. Nature 2002, 418, 823.

31. Barrier, A.; Boelle, P.Y.; Lemoine, A.; Tse, C.; Brault, D.; Chiappini, F.; Lacaine, F.; Houry, S.; Huguier, M.; Flahault, A.; Dudoit, S. Gene expression profiling of nonneoplastic mucosa may predict clinical outcome of colon cancer patients. Dis. Colon Rectum 2005, 48, 2238-2248.

32. Baisse, B.; Bouzourene, H.; Saraga, E.P.; Bosman, F.T.; Benhattar, J. Intratumor genetic heterogeneity in advanced human colorectal adenocarcinoma. Int. J. Cancer 2001, 93, 346-352.

33. Yoshida, T.; Kobayashi, T.; Itoda, M.; Muto, T.; Miyaguchi, K.; Mogushi, K.; Shoji, S.; Shimokawa, K.; Iida, S.; Uetake, H.; Ishikawa, T.; Sugihara, K.; Mizushima, H.; Tanaka, H. Clinical omics analysis of colorectal cancer incorporating copy number aberrations and gene expression data. Cancer Inform 2010, 9, 147-161.

34. Fan, X.; Shi, L.; Fang, H.; Cheng, Y.; Perkins, R.; Tong, W. DNA microarrays are predictive of cancer prognosis: a re-evaluation. Clin. Cancer Res. 2010, 16, 629-636.

35. Bianchini, M.; Levy, E.; Zucchini, C.; Pinski, V.; Macagno, C.; De Sanctis, P.; Valvassori, L.; Carinci, P.; Mordoh, J. Comparative study of gene expression by cDNA microarray in human colorectal cancer tissues and normal mucosa. Int. J. Oncol. 2006, 29, 83-94.

36. Arango, D.; Laiho, P.; Kokko, A.; Alhopuro, P.; Sammalkorpi, H.; Salovaara, R.; Nicorici, D.; Hautaniemi, S.; Alazzouzi, H.; Mecklin, J.P.; Järvinen, H.; Hemminki, A.; Astola, J.; Schwartz, S., Jr.; Aaltonen, L.A. Gene-expression profiling predicts recurrence in Dukes' C colorectal cancer. Gastroenterology 2005, 129, 874-884.

37. Eschrich, S.; Yang, I.; Bloom, G.; Kwong, K.Y.; Boulware, D.; Cantor, A.; Coppola, D.; Kruhøffer, M.; Aaltonen, L.; Orntoft, T.F.; Quackenbush, J.; Yeatman, T.J. Molecular staging for survival prediction of colorectal cancer patients. J. Clin. Oncol. 2005, 23, 3526-3535.

38. Bertucci, F.; Salas, S.; Eysteries, S.; Nasser, V.; Finetti, P.; Ginestier, C.; Charafe-Jauffret, E.; Loriod, B.; Bachelart, L.; Montfort, J.; Victorero, G.; Viret, F.; Ollendorff, V.; Fert, V.; Giovaninni, M.; Delpero, J.R.; Nguyen, C.; Viens, P.; Monges, G.; Birnbaum, D.; Houlgatte, R. Gene expression profiling of colon cancer by DNA microarrays and correlation with histoclinical parameters. Oncogene 2004, 23, 1377-13791.

(C) 2011 by the authors; licensee MDPI, Basel, Switzerland. This article is an open-access article distributed under the terms and conditions of the Creative Commons Attribution license (http://creativecommons.org/licenses/by/3.0/). 